\title{
Cultivo del coral cuerno de alce Acropora palmata en un sistema recirculado utilizando agua de mar sintética
}

\author{
Culture of elkhorn coral Acropora palmata in a recirculating system using synthetic seawater
}

\author{
Galdy Hernández-Zárate ${ }^{1}$, Fabiola Lango-Reynosoํㅜ, María del Refugio Castañeda- \\ Chávez ${ }^{1}$, Jorge E. Zamora-Castro ${ }^{1}$ y Juan Raymundo Maldonado-Hernández ${ }^{2}$
}

\author{
${ }^{1}$ Laboratorio de Investigación de Recursos Acuáticos, Instituto Tecnológico de Boca del Río (ITBOCA), Km 12, Carretera \\ Veracruz-Córdoba, C. P. 94290, Boca del Río, Veracruz, México. fabiolalango@yahoo.com.mx \\ ${ }^{2}$ Corallium, S. A. de C.V. Privada de Azaleas 16, Col. Jardines de Las Ánimas, C. P. 91190, Xalapa, Veracruz, México
}

\begin{abstract}
This study assessed the survival of Acropora palmata fragments under controlled experimental conditions over a period of four months in a recirculating artificial seawater system. The coral fragments $(5-9 \mathrm{~cm}$ long) were fixed in aragocrete and placed in an aquarium under a photoperiod of $12 \mathrm{~h}$ light: $12 \mathrm{~h}$ dark, using 150 W lamps $(10000 \mathrm{~K})$. The survival rate was $58 \%$. Fragment mortality occurred mostly during the first month, which was associated to the process of bleaching stress as a result of fragmentation. These results showed that the conditioning in captivity of $A$. palmata using artificial seawater has potential as a viable tool to help the conservation of this species.
\end{abstract}

Key words: Aquarium, conditioning, fragmentation, stony corals

\section{INTRODUCCIÓN}

En México, el coral cuerno de alce Acropora palmata (Lamarck, 1816) es una de las especies coralinas de mayor importancia debido a que constituye el elemento primario en la formación de arrecifes coralinos en el Golfo de México y el mar Caribe (Aronson \& Precht 2001, SEMARNATCONANP 2008). Esta especie se caracteriza por ser vulnerable a factores de perturbación local como son el desarrollo de actividades turísticas y recreativas, el encallamiento de buques de carga, los eventos climatológicos extremos como huracanes, la introducción de diversos contaminantes agroindustriales, el vertido de agua de lastre, petróleo y grasas, y la extracción no regulada de corales como materiales de ornato o para la construcción (Tunnell 1992, Richmond 1993, Chabanet et al. 2005), además de los efectos del cambio climático que ocurren a nivel global y la presencia de enfermedades infecciosas (Aronson \& Precht 2001). Durante las últimas tres décadas, la combinación de estos factores ha contribuido significativamente al deterioro de los arrecifes de coral ocasionando una disminución en la cobertura de esta especie (50-100\%), sin que se haya observado una recuperación significativa de la misma. Debido a ello, desde 1994, A. palmata se encuentra en la lista de especies amenazadas con protección especial por la Secretaria de Medio Ambiente y Recursos Naturales (NOM-059-ECOL$2001)^{1}$, siendo necesario propiciar la recuperación y conservación de sus poblaciones y sus hábitats.

Una propuesta reciente y prioritaria para la conservación de los corales es su cultivo en cautiverio (Schlacher 2007), a través de la acuacultura ornamental marina, ya que sólo una fracción mínima de los corales $(0,03 \%)$ utilizados como suministro para el comercio de acuarios ornamentales es producida en cautiverio (Green \& Shirley 1999). El conocimiento y mejoramiento de las prácticas y condiciones del cultivo ex situ, así como de propagación y micropropagación, permitirían reducir la captura de especímenes silvestres y el impacto ecológico que conlleva esta actividad. Por lo tanto, es de gran importancia realizar estudios de los parámetros de acondicionamiento de estos organismos, así como realizar la transferencia tecnológica y de conocimientos de centros e institutos de investigación hacia las empresas que se

${ }^{1} \mathrm{NOM}$-059-ECOL-2001. Protección ambiental-Especies nativas de México de flora y fauna silvestres-categorías de riesgo y especificaciones para su inclusión, exclusión o cambio. Lista de especies en riesgo. Secretaría de Medio Ambiente y Recursos Naturales. Diario Oficial de la Federación. 2002. Poder Ejecutivo Federal. Secretaria del Medio Ambiente y Recursos Naturales. México, D.F. miércoles 6 de marzo de 2002. [en línea] <www.semarnat.gob.mx/leyesynormas/Normas\%200ficiales\%20Mexicanas\%20vigentes/NOM-ECOL-059-2001.pdf> 
dedican al cultivo y venta de corales ornamentales. Con base a lo anterior, el objetivo de este trabajo fue acondicionar fragmentos de Acropora palmata para su propagación bajo condiciones de laboratorio, como una etapa preliminar de un proyecto orientado al desarrollo y escalamiento de tecnologías para la restauración y recuperación de hábitats impactados.

\section{Materiales y Métodos}

En abril de 2009, se colectaron cinco colonias madre de Acropora palmata (ca., 10-15 cm de diámetro) frente a las playas de Paso Doña Juana y Chachalacas en el Municipio de Úrsulo Galván $\left(19^{\circ} 27^{\prime} 41,8^{\prime \prime} \mathrm{N}\right.$ y $96^{\circ} 19^{\prime} 48,7$ '”), en el estado de Veracruz, México, fuera del área natural protegida Parque Nacional Sistema Arrecifal Veracruzano. Durante la colecta se registraron los parámetros fisicoquímicos del medio marino para el acondicionamiento de los corales en el acuario.

En el laboratorio, las colonias se aclimataron por la técnica de goteo utilizando agua de mar sintética (Coralife) hasta igualar a una temperatura de $24-26^{\circ} \mathrm{C}$ y una salinidad de 34-35 ups, condiciones similares a las encontradas en su hábitat natural. Los corales se mantuvieron durante un periodo de dos meses previo a la fragmentación, en el área de cuarentena para su observación, revisión y acondicionamiento. Toda manipulación se realizó utilizando guantes para evitar cualquier tipo de contaminación microbiológica y/o química por contacto.

Un total de 26 fragmentos de $c a$., 5 a $9 \mathrm{~cm}$ de longitud x 1-2 cm de ancho, se obtuvieron de las colonias madre mediante la técnica de corte de ramas. Los fragmentos se fijaron en un soporte de agarocreto (aragonita y concreto blanco) de 4,0 $\mathrm{cm}$ de diámetro, utilizando una resina comercial de cianocrilato (Maxi-Cure, Cue Components, FL). Posteriormente se colocaron en un acuario de $c a$. $120 \mathrm{~L}$ de volumen útil, equipado de un rebosadero, un filtro colector (sump) con calcetín y fraccionador de proteínas 'skimmer' (Sander Power Skimmer KA-WT 450), $\mathrm{y}$ un filtro biológico constituido de roca viva, grava y aragonita. El agua de mar sintética se enriqueció incorporando elementos traza como boro, magnesio, molibdeno y estroncio siguiendo las indicaciones del proveedor (Essential elements, Kent Marine). Otros productos utilizados para proveer los requerimientos de calcio de los fragmentos de coral fueron: Reef calcium ${ }^{\mathrm{TM}}$, Reef builder ${ }^{\mathrm{TM}}$, Reef advantage calcium ${ }^{\mathrm{TM}}$, Reef advantage magnesium $^{\mathrm{TM}}$, Reef complete $^{\mathrm{TM}}$ y Reef plus ${ }^{\mathrm{TM}}$ de la marca Seachem. Debido a que Acropora palmata es una especie que habita en aguas someras de alta irradiancia, el sistema de propagación se acopló con dos lámparas temporizadas de haluro metálico ( $400 \mu \mathrm{mol}$ fotones $\mathrm{m}^{-2} \mathrm{~s}^{-1}, 150 \mathrm{~W}, 10000$ $\mathrm{K}$ ), colocadas a una altura de $25 \mathrm{~cm}$ sobre el nivel del agua para simular la luz de día y una lámpara tipo LED (Current, Modelo 1042) para simular la luz de luna, manteniendo un fotoperiodo de $12 \mathrm{~h}$ luz: $12 \mathrm{~h}$ oscuridad.

La alimentación de los fragmentos se realizó dos veces por semana. Una de ellas, con una mezcla comercial (Phytomax, Kent Marine) de fitoplancton (Nannochloropsis, Isochrysis y Tetraselmis) y un suplemento alimenticio con proteínas, omega-3 y ácidos grasos omega-6, fitonutrientes, carotenoides, vitaminas y minerales (ChromaMax, Kent Marine); y la otra con zooplancton (Artemia) comercial (Zoomax, Kent Marine).

Diariamente, durante un periodo de cuatro meses, se realizó una inspección visual de los organismos para registrar: aparición de blanqueamiento y secreción de mucosa. Además, se determinó el porcentaje de supervivencia $\% \mathrm{~S}=(\Sigma \mathrm{nf} / \Sigma \mathrm{ni}) 100$, donde $\mathrm{S}$ es la supervivencia (\%), $\Sigma$ nf es el número final de organismos y $\Sigma$ ni es el número inicial de organismos. Los parámetros fisicoquímicos del agua fueron registraron dos veces por semana. La temperatura y el $\mathrm{pH}$ se midieron con un potenciómetro (YSI Mod. pH 10), y la salinidad con un refractómetro portátil (Captive purity refractometer) con escala de 0 a 100 unidades prácticas de salinidad (ups). Las concentraciones de amonio, nitratos y fosfatos se analizaron mediante métodos colorímetros utilizando las pruebas de la casa comercial $\mathrm{HACH}^{\circledR}$. La alcalinidad y el calcio se analizaron mediante métodos comerciales (Nutrafin Calcium Test $\mathrm{Kit}^{\mathrm{TM}}$ y Nutrafin Alkalinity Tes kitt $^{\mathrm{TM}}$, Hagen, Inc.). Para detectar diferencias entre medias de las variables fisicoquímicas entre meses, se realizó un análisis de rangos múltiples Kruskal-Wallis con un nivel de confianza del 95\% $(\alpha=0,05)$. Todos los análisis se realizaron mediante el programa estadístico STATISTICA (StatSoft, versión 7,0).

\section{Resultados y Discusión}

La salinidad, en el sistema de cultivo de Acropora palmata, se mantuvo dentro de los valores típicos del agua de mar, oscilando entre 34,2 y 35,3 ups (Fig. 1A). Estos valores se encontraron dentro del rango óptimo de salinidad para el género Acropora (34-37 ups) (Boulon et al. 2005), y son acordes a las concentraciones recomendadas por Watson \& Hills (2006) para el cultivo de corales en sistemas recirculados ( 33 y 35 ups). La temperatura del agua recibió una atención especial por considerarse un factor crítico para el crecimiento y 

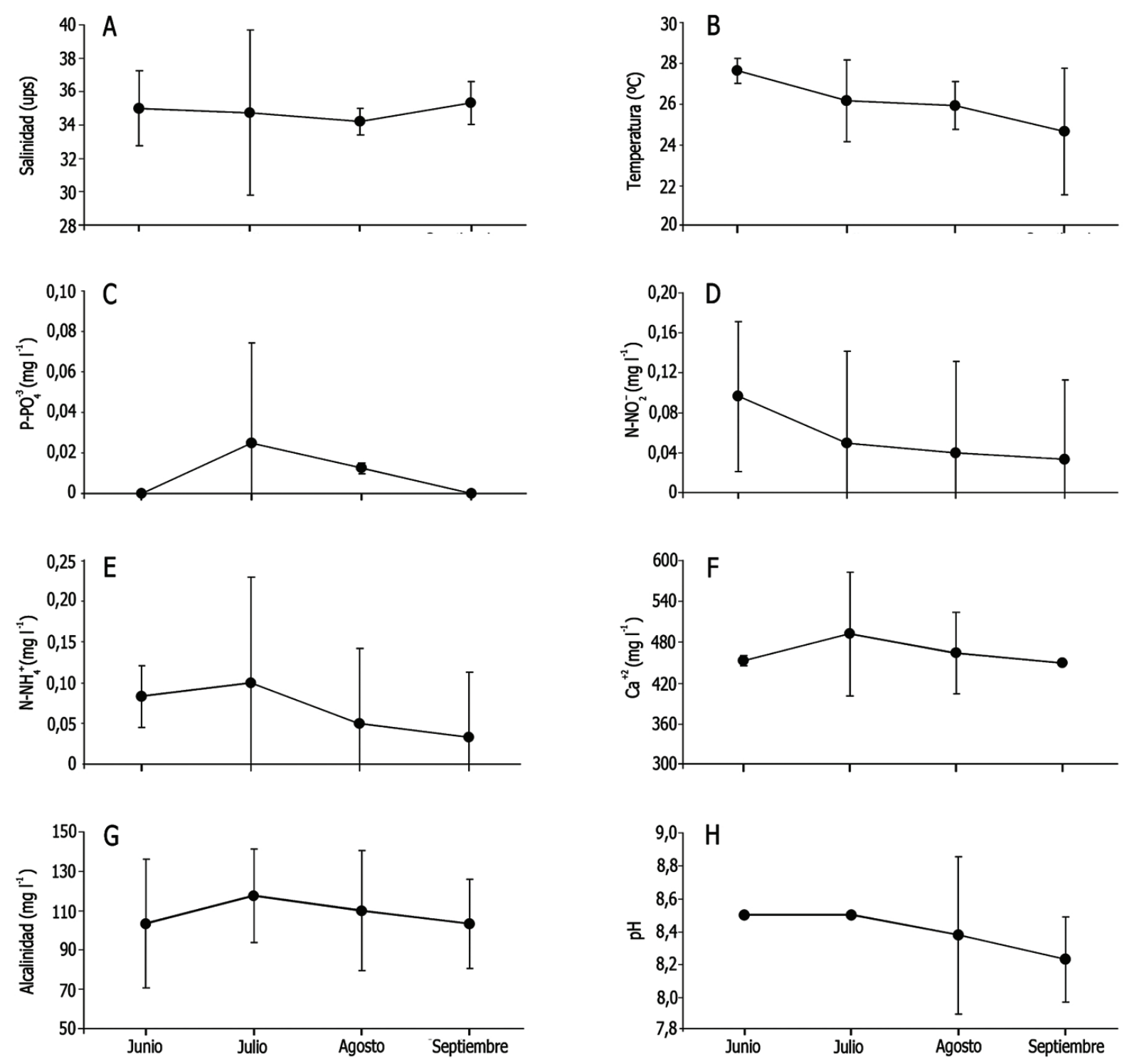

Figura 1. Valores promedio de los parámetros fisicoquímicos en el sistema recirculado de Acropora palmata. A: salinidad, B: temperatura, C: fosfato, D: nitritos, E: amonio, F: calcio, G: alcalinidad, H: pH. Las barras verticales representan el intervalo de confianza al 95\% / Mean concentration of physicochemical parameters in the recirculating system of Acropora palmata. A: salinity, B: temperature, C: phosphates, D: nitrite, E: ammonium, F: calcium, G: alkalinity, $\mathrm{H}: \mathrm{pH}$. Vertical bars represent $95 \%$ confidence intervals

reproducción de los corales (Baird \& Marshall 2002). Este parámetro se mantuvo en el rango óptimo de $A$. palmata $\left(25\right.$ a $\left.29^{\circ} \mathrm{C}\right)$ (Jaap et al. 1988 , Boulon et al. 2005) para evitar el blanqueamiento por estrés térmico (Brown 1997). El valor mayor se registró en el mes de junio $\left(27,4^{\circ} \mathrm{C}\right)$ y el menor en el mes de septiembre $\left(24,7^{\circ} \mathrm{C}\right)$, con un promedio general de $26,1 \pm 1,2^{\circ} \mathrm{C}$ (Fig. 1B). Un descenso gradual de la temperatura se llevó a cabo después del mes de junio, con el fin de favorecer la producción de zooxantelas e incrementar la eficiencia fotosintética (Metalpa et al. 2008). Esta variación no fue estadísticamente significativa entre los meses evaluados $\left(\mathrm{H}_{(3,9)}=6,95, P=0,07\right)$.

En relación a los nutrientes, los fosfatos se encontraron por debajo de $0,03 \mathrm{mg} \mathrm{L}^{-1}$ (Fig. 1C), mientras las concentraciones de nitritos y amonio oscilaron entre 0,03 y $0,10 \mathrm{mg} \mathrm{L}^{-1}$, para ambos casos (Figs. 1D y E). Es bien conocido que en acuarios y sistemas recirculados, la mayoría de las especies ornamentales marinas presentan 
una tolerancia baja al amonio y a los nitritos, por lo que diversos autores recomiendan mantenerlos en niveles cercanos a cero $\left(0,004\right.$ a $\left.0,1 \mathrm{mg} \mathrm{L}^{-1}\right)$ (Wajsbrot et al. 1993, Watson \& Hill 2006). No obstante, en los corales escleractinios hermatípicos, el nitrógeno y el fósforo son requeridos en bajas concentraciones $\left(<1,0 \mathrm{mg} \mathrm{N}^{-N_{4}}{ }_{4}^{+} \mathrm{l}^{-}\right.$ y $0,2 \mathrm{mg} \mathrm{P}-\mathrm{PO}_{4}^{-3} \mathrm{~L}^{-1}$, respectivamente) por las zooxantelas simbiontes, siendo transformados junto con el dióxido de carbono atmosférico en nutrientes disponibles para el coral (Szmant 2002, Baohua et al. 2004). Niveles de nutrientes mayores a los encontrados en este trabajo, tienen efectos significativos sobre la fisiología de los corales, tales como la disminución de la tasa de crecimiento y de reproducción o el aumento en la susceptibilidad a la decoloración (Szmant 2002). Sin embargo, el efecto más importante en los corales escleractinios es la reducción de la tasa de calcificación debido al enriquecimiento del fósforo (más que por amonio), el cual limita la extensión de la estructura esquelética (Stambler et al. 1991). El bajo contenido de fosfatos y compuestos nitrogenados, indicaron un buen estado de la calidad del agua para el cultivo de Acropora palmata en cautiverio.

Otro de los elementos primordiales en la formación de los corales es el carbonato de calcio $\left(\mathrm{CaCO}_{3}\right)$, el cual se mantuvo en el rango de 450 a $492,5 \mathrm{mg} \mathrm{L}^{-1}$ (Fig. 1F). El calcio es utilizado por los corales pétreos para formar sus esqueletos. Este proceso de calcificación es dependiente de la luz y de la temperatura (Kajiwara et al. 1995). Reynaud et al. 2004 demostraron que la tasa de crecimiento de Acropora verweyi fue significativamente mayor bajo intensidades de luz $\left(400 \mu \mathrm{mol} \mathrm{m} \mathrm{m}^{-2} \mathrm{~s}^{-1}\right)$ y de temperatura $\left(29^{\circ} \mathrm{C}\right)$ altas. En este contexto, para favorecer el proceso de calcificación de $A$. palmata, el sistema se acopló con una fuente de luz de alta intensidad $(10000 \mathrm{~K})$, manteniendo los niveles de calcio cercanos a los encontrados en el agua de mar del sitio de extracción $\left(530,0 \pm 7,8 \mathrm{mg} \mathrm{L}^{-1}\right)$.

La alcalinidad fluctuó en función de la concentración de calcio (Figs. 1F y G), permaneciendo por encima de los $100 \mathrm{mg} \mathrm{L}^{-1}$, lo que indica que los productos comerciales utilizados en este estudio mostraron una buena capacidad amortiguadora, al mantener la alcalinidad del sistema. El uso de roca viva en el biofiltro, también pudo jugar un rol importante en la dinámica de los carbonatos, manteniendo el equilibrio entre carbonatos/bicarbonatos (Yuen et al. 2009). Por su parte, el pH se mantuvo por encima de 8,2 (Fig. 1H), condición que posiblemente favoreció el proceso de calcificación de los corales, al existir una mayor disponibilidad de carbonatos (Marubini et al. 2008).
El uso de un filtro biológico construido con roca viva permitió mantener las concentraciones de amonio y nitritos en niveles seguros para Acropora palmata, lo cual coincide con lo señalado por Yuen et al. (2009). Además, el fraccionador de proteínas redujo las fuentes de nitrógeno orgánico disuelto y/o en suspensión generados por desechos procedentes del alimento no digerido por el coral o procedentes del tejido muerto (microalgas y pólipos).

No se evidenció diferencias significativas en las concentraciones promedio de la salinidad $\left(\mathrm{H}_{(3,9)}=3,01, P\right.$ $=0,39), \mathrm{pH}\left(\mathrm{H}_{(3,9)}=6,50, P=0,09\right)$, fosfatos $\left(\mathrm{H}_{(3,9)}=1,96\right.$, $P=0,58)$, nitritos $\left(\mathrm{H}_{(3,9)}=1,23, P=0,75\right)$, amonio $\left(\mathrm{H}_{(3,9)}=\right.$ $3,27, P=0,35)$, alcalinidad $\left(\mathrm{H}_{(3,9)}=1,70, P=0,64\right)$ y calcio $\left(\mathrm{H}_{(3,9)}=2,84, P=0,42\right)$ entre los meses evaluados.

La mortalidad de corales (fragmentos considerados como muertos) fue de $42 \%$, siete fragmentos (de $n=11$ ) murieron durante el primer mes, evento asociado al proceso de blanqueamiento como resultado del estrés por fragmentación y la manipulación que esto implica. Durante este periodo los organismos mostraron abundante secreción de mucosa, y posteriormente la aparición de blanqueamiento, acompañado de la pérdida de tejido. Luego del blanqueamiento, los fragmentos murieron en un periodo de aproximadamente siete días. Estos resultados coinciden con Jones (2008), quien en estudios realizados in situ observó que la mortalidad en Acropora spp., estuvo íntimamente ligada a eventos de blanqueamiento (pérdida de zooxantelas endosimbióticas), posiblemente por agentes infecciosos o estrés ambiental (Brown 1997, Aronson \& Precht 2001, Baird \& Marshall 2002). Después del mes de junio, la mortalidad decreció gradualmente, muriendo tres fragmentos en el mes de julio y uno en el mes de agosto, también por blanqueamiento. Para el mes de septiembre no hubo mortalidad. Básicamente, después de un periodo de experimentación de cuatro meses de cultivo, la tasa de supervivencia para Acropora palmata fue del $58 \%(\mathrm{n}=15)$. Estos valores fueron similares a los encontrados por Schlacher et al. (2007) quienes encontraron un 52\% de supervivencia en Acropora solitaryensis y Yuliantri et al. (2006) quienes mostraron un rango de 42,86-85,71\% en Acropora microphthalma.

Algunos autores han indicado que fragmentos de menor tamaño son más susceptibles a la mortalidad por fragmentación, ya que los de mayor tamaño poseen más reservas energéticas y pueden recuperarse más fácilmente del estrés (Oren et al. 2001, Okubo et al. 2007). Sin embargo, en este estudio, de los 11 fragmentos que 
murieron por blanqueamiento, 6 de ellos $(54,5 \%)$ presentaron una longitud menor a los $6,8 \mathrm{~cm}$, mientras que los 5 restantes $(45,5 \%)$ se encontraron en una longitud mayor a los 7,6 cm. Resultados similares también fueron observados por Lirman (2000).

Muchos corales poseen algas simbiontes zooxantelas, y la luz es un factor crítico para el buen estado de salud de los corales (Anthony \& Hoegh-Guldberg 2003). En ambientes naturales, el rol de la luz ha sido bien estudiado (Falkowski et al. 1984, Anthony \& Fabricius 2000). Por el contrario, la optimización de las fuentes de luz en la industria de los acuarios es un tema que continua pobremente documentado (Calfo 2001). En este trabajo se utilizaron lámparas de 10000 Kelvin (luz roja), debido a que Acropora palmata es una especie que tolera una mayor intensidad de luz que otras especies de coral que habitan en aguas profundas (SEMARNAT-CONANP 2008), sin embargo, aún se requiere realizar estudios que permitan determinar la fuente de luz artificial optima (intensidad, régimen de luz y composición espectral, entre otras) para favorecer el crecimiento y la supervivencia de A. palmata en cautiverio.

En conclusión, se obtuvo la viabilidad en el procedimiento de colecta, transplante y acondicionamiento de Acropora palmata para su propagación en cautiverio utilizando agua de mar sintética. La utilización de estos sistemas puede contribuir al uso y aprovechamiento sustentable de los recursos marinos, como alternativa a la extracción del hábitat, favoreciendo así la conservación de especies y el repoblamiento.

\section{Agradecimientos}

Este trabajo se financió por el Consejo Nacional de Ciencia y Tecnología de México, como parte del proyecto PROINNOVA 'Integración de un Paquete Tecnológico para el Cultivo-Propagación de Fauna Silvestre' (CONACYT-Proyecto 140439). Se agradece al grupo técnico de la empresa Corallium ${ }^{\mathrm{TM}} \mathrm{S}$. A. de C. V. por el apoyo brindado para la realización de este trabajo.

\section{LITERATURA CITADA}

Anthony KRN \& KE Fabricius. 2000. Shifting roles of heterotrophy and autotrophy in coral energetics under varying turbidity. Journal of Experimental Ecology 252: 221-253.

Anthony KRN \& O Hoegh-Guldberg. 2003. Variation in coral photosynthesis, respiration and growth characteristics in contrasting light microhabitats: an analogue to plants in forest gaps and understoreys? Functional Ecology 17: 246259.
Aronson RB \& WF Precht. 2001. White-band disease and the changing face of Caribbean coral reefs. Hydrobiologia 460: 25-38.

Baird AH \& PA Marshall. 2002. Mortality, growth and reproduction in scleractinian corals following bleaching on the Great Barrier Reef. Marine Ecology Progress Series 237: $133-141$

Baohua Z, W Guangce, H Bo \& CK Tseng. 2004. Effects of temperature, hypoxia, ammonia and nitrate on the bleaching among three coral species. Chinese Science Bulletin 49(18): 1923-1928.

Boulon R, M Chiappone, R Halley, W Jaap, B Keller, B Kruczynski, M Miller \& C Rogers. 2005. Atlantic Acropora status review document. Acropora Biological Review Team. Report to National Marine Fisheries Service, Southeast Regional Office, Miami, 152 pp.

Brown BE. 1997. Coral bleaching: causes and consequences. Coral Reefs 16: 129-138.

Calfo RA. 2001. Book of coral propagation. Reef gardening for Aquarists 1: 1-450. Reading Trees Publications, Pennsylvania.

Chabanet P, M Adjeroud, S Andréfouët, YM Bozec, J Ferraris, JA Garcìa-Charton \& M Schrimm. 2005. Human-induced physical disturbances and their indicators on coral reef habitats: A multi-scale approach. Aquatic Living Resources 18: 215-230.

Falkowski PG, Z Dubinsky, L Muscatine \& JW Porter. 1984. Light and the bioenergetics of a symbiotic coral. Bioscience 34: 705-709.

Green E \& F Shirley. 1999. The global trade in corals. WCMC Biodiversity Series 10: 1-74, World Conservation Monitoring Centre, Cambridge.

Jaap WC, JC Halas \& RG Muller. 1988. Community dynamics of stony corals (Scleractinia and Milleporina) at the Key Largo National Marine Sanctuary, Key Largo, Florida during 1981-1986. Proceedings of the 6th International Coral Reef Symposium 2: 237-243.

Jones RJ. 2008. Coral bleaching, bleaching-induced mortality, and the adaptive significance of the bleaching response. Marine Biology 154: 65-80.

Kajiwara K, A Nagai \& S Ueno. 1995. Examination of the effect of temperature, light intensity and zooxanthellae concentration on calcification and photosynthesis of scleractinian coral Acropora pulchra. Journal of the School of Marine Science and Technology 40: 95-103.

Lirman D. 2000. Lesion regeneration in the branching coral Acropora palmata: effect of colonization, colony size, lesion size, and lesion shape. Marine Ecology Progress Series 17: 209-215. 
Marubini F, C Ferrier-Page's, P Furla \& D Allemand. 2008. Coral calcification responds to seawater acidification: a working hypothesis towards a physiological mechanism. Coral Reefs 27: 491-499.

Metalpa RR, A Peirano, F Houlbrè, M Abbate \& C FerrierPagès. 2008. Effects of temperature, light and heterotrophy on the growth rate and budding of the temperate coral Cladocora caespitosa. Coral Reef 27: 17-25.

Okubo N, T Motokawa \& M Omori. 2007. When fragmented coral spawn? Effect of size and timing on survivorship and fecundity of fragmentation in Acropora formosa. Marine Biology 151: 353-363.

Oren U, Y Benayahu, H Lubinevsky \& Y Loya. 2001. Colony integration during regeneration in the Stony coral Favia favus. Ecology 82(3): 802-813.

Reynaud S, C Ferrier-Pagès, F Boisson, D Allemand \& RG Fairbanks. 2004. Effect of light and temperature on calcification and strontium uptake in the scleractinian coral Acropora verweyi. Marine Ecology Progress Series 279: 105-112.

Richmond RH. 1993. Coral reefs: present problems and future concerns resulting from anthropogenic disturbance. American Zoologist 33: 524-536.

Schlacher TA, J Stark \& ABP Fischer. 2007. Evaluation of artificial light regimes and substrate types for aquaria propagation of the staghorn coral Acropora solitaryensis. Aquaculture 269: 278-289.

SEMARNAT-CONANP. 2008. Programa de acción para la conservación de las especies: corales cuerno de ciervo (Acropora cervicornis) y cuerno de alce (Acropora palmata) (PACE: Acroporas). [en línea] <http://www.conanp.gob.mx/ pdf_especies/PACE\%20Acroporas\%20-1.pdf>
Stambler N, N Popper, Z Dubinsky \& J Stimson. 1991. Effects of nutrient enrichment and water motion on the coral Pocillopora damicornis. Pacific Science 45: 299-807.

Szmant AM. 2002. Nutrient enrichment on coral reefs: Is it a major cause of coral reef decline? Estuaries 25(4b): 743766.

Tunnell JW Jr 1992. Natural versus human impacts to Southern Gulf of Mexico coral-reef resources. Proceedings of the 7th International Coral Reef Symposium Guam 1: 300-306.

Wajsbrot N, A Gasith, A Diamant \& DM Popper. 1993. Chronic toxicity of ammonia to juvenile gilthead seabream Sparus aurata and related histopathological effects. Journal of Fish Biology 42: 321-328.

Watson CA \& JE Hill. 2006. Design criteria for recirculating, marine ornamental production systems. Aquaculture Engineering 34: 157-162.

Yuen YS, SS Yamazaki, T Nakamura, G Tokuda \& H Yamasaki. 2009. Effects of live rock on the reef-building coral Acropora digitifera cultured with high levels of nitrogenous compounds. Aquacultural Engineering 41(1): $35-43$.

Yuliantri AR, W Moka, J Jompa \& M Litaay. 2006. The successful transplantation of Acropora microphthalma at Barrang Lompo Reef Edge, South Sulawesi. Marine Research in Indonesia 30: 21-25.

Recibido el 28 de diciembre de 2010 y aceptado el 17 de octubre de 2011 